\title{
A Mixed Finite Element Formulation for Elastoplasticity
}

\author{
M. Nagler, A. Pechstein and A. Humer \\ Institute of Technical Mechanics \\ Johannes Kepler University Linz \\ Altenbergerstraße 69, 4040 Linz, Austria \\ e-mail: michaela.nagler@jku.at
}

\begin{abstract}
Efficient simulation of thin-walled structures, whose thickness can be orders of magnitude smaller than the in-plane dimensions, is essential in the analysis of sheet metal forming processes, for example. Discretizing thin structures with standard finite element methods is challenging due to diverse locking phenomena. Additionally, the elastoplastic material behavior adds even more complexity and difficulties. In the present contribution, we propose a variational formulation for elastoplasticity, on which we build a novel mixed finite element formulation. Our approach is based on a HellingerReissner type formulation that uses the tangential component of the displacement field and the normal component of the normal stress vector as degrees of freedom. This tangential-displacement normalnormal-stress (TDNNS) method provide elements, which do not suffer from shear locking when discretizing thin structures $[1,2]$ for problems in linear elasticity. In the current contribution, the TDNNS method is extended from linear elasticity to plasticity. Instead of using the classical returnmapping algorithm [2], a variational inequality is derived within a consistent thermodynamical formulation based on the second law of thermodynamics. For the elastoplastic material a von Mises yield criterion, which can expand isotropically and translate kinematically in stress space, is considered. Rather than starting from the definition of a yield function and a flow potential, the definition of the dissipation inequality and a corresponding dissipation function is considered. With the TDNNS method, we can reduce the number of degrees of freedom significantly as compared to conventional approaches. For validation purposes, our numerical results in several benchmark problems are compared to results obtained with the commercial finite element code Abaqus.
\end{abstract}

\section{REFERENCES}

[1] A. Pechstein and J.Schöberl, Tangential-displacement and normal-normal-stress continuous mixed finite elements for elasticity, Math. Models Methods Appl. Sci. 21, (8) (2011), pp. 17611782. doi:10.1142/S0218202511005568.

[2] A. Pechstein and J.Schöberl, Anisotropic mixed finite elements for elasticity, Int. J. Numer. Methods Eng. 90 (2) (2012), pp. 196-217. doi:10.1002/nme.3319.

[3] J. C. Simo and T. JR. Hughes, Computational inelasticity, volume 7. Springer Sciece \& Business Media, 2006 